\title{
Phase Domain Walls in Weakly Nonlinear Deep Water Surface Gravity Waves
}

\author{
F. Tsitoura, ${ }^{1}$ U. Gietz, ${ }^{2}$ A. Chabchoub ${ }^{3}$ and N. Hoffmann ${ }^{1,4}$ \\ ${ }^{1}$ Dynamics Group, Hamburg University of Technology, Hamburg 21073, Germany \\ ${ }^{2}$ Department of Fluid Dynamics and Ship Theory, Hamburg University of Technology, Hamburg 21073, Germany \\ ${ }^{3}$ School of Civil Engineering, The University of Sydney, Sydney, NSW 2006, Australia \\ ${ }^{4}$ Department of Mechanical Engineering, Imperial College London, London SW7 2AZ, United Kingdom
}

(Received 22 June 2017; published 1 June 2018)

\begin{abstract}
We report a theoretical derivation, an experimental observation and a numerical validation of nonlinear phase domain walls in weakly nonlinear deep water surface gravity waves. The domain walls presented are connecting homogeneous zones of weakly nonlinear plane Stokes waves of identical amplitude and wave vector but differences in phase. By exploiting symmetry transformations within the framework of the nonlinear Schrödinger equation we demonstrate the existence of exact analytical solutions representing such domain walls in the weakly nonlinear limit. The walls are in general oblique to the direction of the wave vector and stationary in moving reference frames. Experimental and numerical studies confirm and visualize the findings. Our present results demonstrate that nonlinear domain walls do exist in the weakly nonlinear regime of general systems exhibiting dispersive waves.
\end{abstract}

DOI: $10.1103 /$ PhysRevLett.120.224102

Domain walls are transition zones of finite width between neighboring homogeneous domains. They are of fundamental importance in many fields of modern physics. In wave dynamics, domain walls connecting neighboring wave fields differing in wave amplitude and phase are also called wave jumps [1-6].

The question of the existence of domain walls between finite amplitude surface gravity waves on deep water, i.e., between domains of deep water Stokes waves, has been investigated at least since the 1960s [7-10]. The dispersive nature of water waves shows that shape conserving domain walls do not exist in the linear limit of infinitesimally small wave amplitudes. However, already early on there was speculation about the existence of weakly nonlinear domain walls in the small but nonzero amplitude limit, where nonlinearity might counteract dispersion and might lead to the existence of shape conserving wall or jump type behavior. While similar considerations in the context of localization have led to the discovery of solitons and breathers $[11,12]$ in weakly nonlinear waves, there were only a few attempts to derive and study analogous shape conserving solutions for domain walls or wave jumps.

Only recently a remarkable series of experimental and theoretical studies has sparked new interest in the field [13-15]. By performing wave tank tests a wave jump between neighboring domains of fully out of phase Stokes waves has been studied. The authors introduced both scalar and coupled systems of nonlinear Schrödinger equations to explain the observed wave state.

The present study has been motivated by these results but starts from a different, simpler conceptual perspective. We employ a $2 \mathrm{D}+1$ (D stands for the spatial dimensions) focusing nonlinear Schrödinger equation (NLS) to obtain analytical solutions for weakly nonlinear domain walls. The NLS is the universal lowest order equation describing the spatiotemporal dynamics of weakly nonlinear narrowbanded wave packets $[16,17]$. The $2 \mathrm{D}+1$ form has been used for various questions on stability and nonplanar solutions [18-21]. In these latter pioneering contributions, the diffraction of Stokes waves that propagate in two dimensions has been studied theoretically, numerically, and experimentally. We emphasize that our domain transition investigated in our study remains stationary in the moving reference frame. The $2 \mathrm{D}+1 \mathrm{NLS}$ equation can be derived by the method of multiple scales [22-24],

$i\left(\frac{\partial A}{\partial t}+c_{g} \frac{\partial A}{\partial x}\right)-\alpha\left(\frac{\partial^{2} A}{\partial x^{2}}-2 \frac{\partial^{2} A}{\partial y^{2}}\right)-\beta|A|^{2} A=0$,

where $A(x, y, t)$ is the complex wave envelope, $t$ denotes time, $x$ and $y$ are the orthogonal horizontal spatial coordinates, with unidirectional background wave propagation along the $x$ axis, and $c_{g}$ is the group velocity. The dispersion and nonlinearity coefficients $\alpha$ and $\beta$ are functions of frequency $\omega$ and wave number $\kappa$, and for deep water waves result as

$$
\alpha=\frac{\omega}{8 \kappa^{2}}, \quad \beta=\frac{\omega \kappa^{2}}{2} .
$$

The linear dispersion relation is $\omega=\sqrt{g \kappa}$, with $g$ the constant of gravity. To first order in wave steepness, the surface elevation $\eta(x, y, t)$ is given as 


$$
\eta(x, y, t)=\operatorname{Re}\{A(x, y, t) \exp [i(\kappa x-\omega t)]\} .
$$

For simplicity, we consider a scaled form of the $2 \mathrm{D}+1$ NLS,

$$
i u_{T}+u_{X X}-2 u_{Y Y}+2|u|^{2} u=0,
$$

which is obtained from Eq. (1) by introducing the scaled variables

$$
\begin{aligned}
X & =x-c_{g} t, & Y & =y, \\
T & =-\alpha t, & u & =\sqrt{\frac{\beta}{2 \alpha}} A .
\end{aligned}
$$

A transformation introduced earlier [25], based on the idea to investigate solutions of the $2 \mathrm{D}+1$ NLS that actually depend on one spatial direction only, allows the derivation of solutions for the $2 \mathrm{D}+1$ NLS from solutions of a $1 \mathrm{D}+1$ NLS. For that purpose we introduce an angle $\gamma$ and a new spatial coordinate $Z$ according to

$$
Z=\frac{X \cos \gamma+Y \sin \gamma}{\sqrt{\left|1-3 \sin ^{2} \gamma\right|}}
$$

We obtain

$$
i \frac{\partial u}{\partial T}+s \frac{\partial^{2} u}{\partial Z^{2}}+2|u|^{2} u=0
$$

which is indeed a $1 \mathrm{D}+1$ NLS, involving a sign factor $s=\left[1-3 \sin ^{2}(\gamma)\right] /\left[\left|1-3 \sin ^{2}(\gamma)\right|\right]$.

A solution of the 1D +1 NLS Eq. (7) thus corresponds to a solution of the 2D +1 NLS Eq. (1). Depending on $\gamma$, two cases result. For small values of $\gamma<35.26^{\circ}, s=+1$ and the 1D $+1 \mathrm{NLS}$ is focusing. For $\gamma>35.26^{\circ}, s=-1$ and the $1 \mathrm{D}+1 \mathrm{NLS}$ turns out defocusing. While the former case has already been studied [26,27], we will consider the latter case here.

For the present purpose of identifying the desired shape conserving stationary or quasistationary domain wall solutions in the $2 \mathrm{D}+1$ focusing NLS, we choose the corresponding shape conserving spatially localized nonlinear solutions of the 1D +1 defocusing NLS: black and gray solitons [28-34], also referred to as dark solitons.

Gray and black solitons of the 1D +1 defocusing NLS possess exact analytical solutions of the form

$$
\begin{aligned}
u(Z, T)= & {[\sin (\theta)+i \cos (\theta) \tanh \{\cos (\theta)[Z-2 \sin (\theta) T]\}] } \\
& \times \exp (2 i T+i \chi) .
\end{aligned}
$$

Dark solitons of the 1D +1 defocusing NLS connect wave domains of identical amplitude which are out of phase. The black soliton, $\theta=0$, represents the case which goes along with a phase change of $\pi$ and drops of the wave envelope to zero, while for gray solitons, $\theta \neq 0$, the amplitude drops and the phase difference is less marked.

With this we can now construct domain wall solutions for the $2 \mathrm{D}+1$ focusing NLS, or small amplitude weakly nonlinear deep water surface gravity Stokes waves. Indeed, we have two free parameters at hand. The first parameter is $\theta$, originating from the one-parameter family of gray or black solitons. The second parameter is $\gamma$, originating from the transformation between 1D and 2D formulations. The carrier wave amplitude $a$ and frequency $\omega$ may be understood as further parameters.

Combining all of the above, the solution of Eq. (8) can be transformed, using the transformations (5) and (6), into a solution of Eq. (1). It may be written, with an additional constant $\chi$, as

$$
\begin{aligned}
A(x, y, t)= & \frac{a}{\sqrt{2} \kappa^{2}}[\sin (\theta)+i \cos (\theta) \tanh \{\cos (\theta) M\}] \\
& \times \exp \left(-2 i a^{2} \alpha t+i \chi\right)
\end{aligned}
$$

where

$$
\begin{aligned}
M= & a\left[\frac{x \cos (\gamma)+y \sin (\gamma)}{\sqrt{\left|1-3 \sin ^{2}(\gamma)\right|}}\right. \\
& \left.+\left(2 a \alpha \sin (\theta)-c_{g} \frac{\cos (\gamma)}{\sqrt{\left|1-3 \sin ^{2}(\gamma)\right|}}\right) t\right] .
\end{aligned}
$$

In the following we will discuss the key properties of the resulting solutions. For simplicity, and ease of illustration, we will compare the results with experimental realizations obtained in a water wave tank.

The experiments have been conducted in a wave facility installed at the Hamburg ship model testing facility (HSVA). The wave tank is shown schematically in Fig. 1. It has dimensions of $50 \times 5 \times 2.4 \mathrm{~m}^{3}$. The wave maker consists of 10 flaps, each with a width of $0.5 \mathrm{~m}$. The paddles are installed at one end of the tank and an absorbing beach of $10 \mathrm{~m}$ is located at the other side.

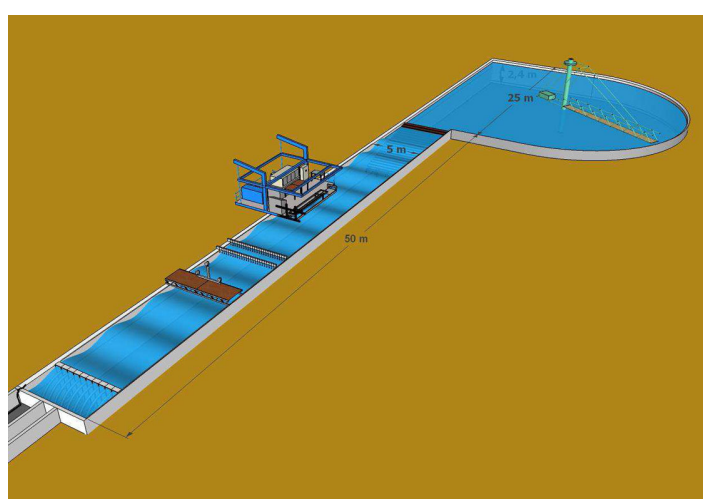

FIG. 1. Schematic illustration of the water wave tank at the Hamburg ship model testing facility. Dimensions are $50 \times 5 \times 2.4 \mathrm{~m}^{3}$. 

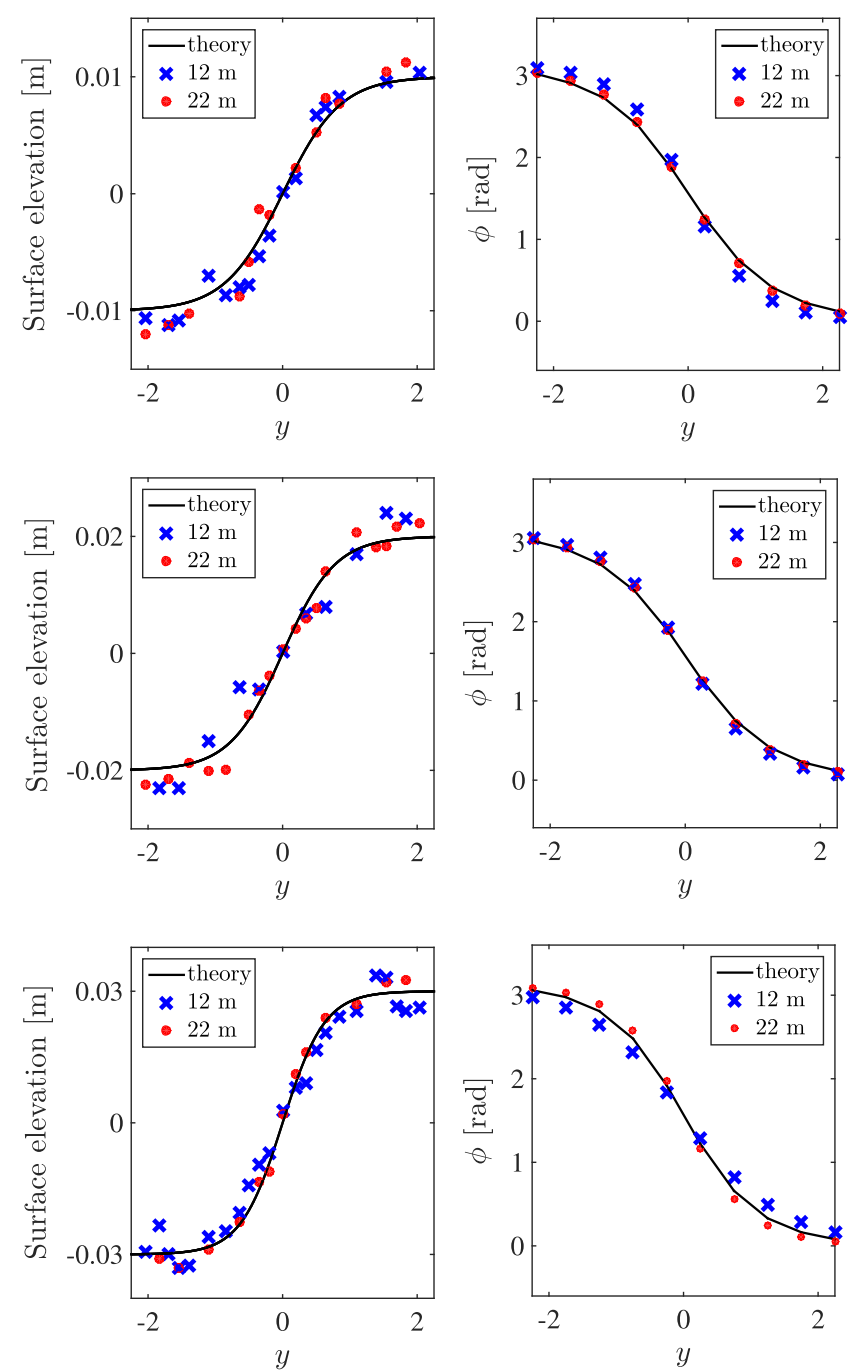

FIG. 2. Surface elevation and phase measured at a distance of 12 and $22 \mathrm{~m}$ [(blue) crosses and (red) dots] from the wave maker. $a \kappa=0.11,0.16$ and 0.22 (upper, middle and lower panels). Solid (black) lines indicate the theoretical curves.

The resulting propagation distance for the waves is about $40 \mathrm{~m}$. Lines of markers are installed at various distances from the wave maker. Each line has 25 markers where the first is centered at the middle of the tank and the others are equally spaced towards the tank wall. The motion of the markers is measured by a VICON (MX-3+) camera system with a sampling frequency of $50 \mathrm{~Hz}$.

We choose an $(x, y)$ coordinate system with $x$ along the tank and $y$ in the transverse direction. The paddles are labeled by an integer $j \in[1,10]$, and in the following $y_{j}$ indicates the transverse location of the center of the $j$ th paddle, assuming all of them located at $x=0$. The timedependent displacement of each paddle can be written in the form of a time-dependent amplitude $a_{j}(t)$ and a timedependent phase $\phi_{j}(t)$,

$$
\eta_{j}\left(a_{j}, y_{j}\right)=\operatorname{Re}\left\{a_{j} \exp \left(i \phi_{j}\right)\right\} .
$$

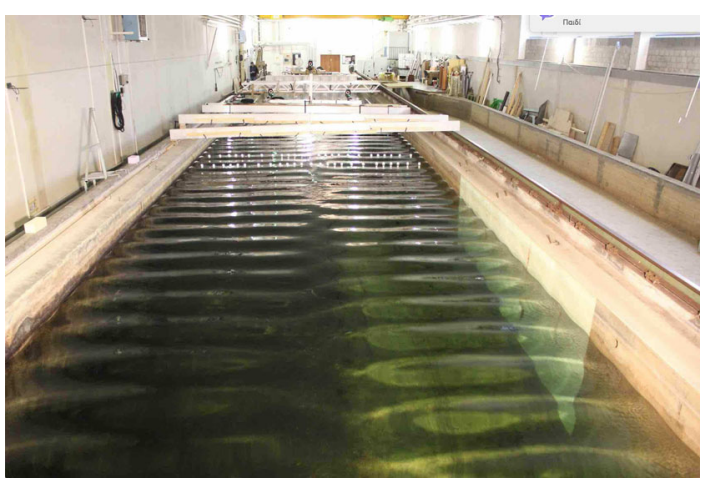

FIG. 3. Photographic image taken at a distance of $35 \mathrm{~m}$ from the wave maker for the experiment presented in the top panel of Fig. 2: $a=0.01 \mathrm{~m} ; \kappa=10.8 \mathrm{~m}^{-1}$.

The motion of the paddles is set according to the domain wall solutions described above. To evaluate the influence of the nonlinearity, all tests have been conducted for varying degrees of wave steepness.

First we discuss the domain wall arising from the black soliton, $\theta=0$, and a transformation angle of $\gamma=\pi / 2$. The paddles move with a phase variation in the $y$ direction corresponding to a hyperbolic tangent.

Three different carrier wave steepness values, $a \kappa=0.11$, $a \kappa=0.16$, and $a \kappa=0.22$ have been tested. All of them are below the breaking threshold of Stokes waves [35]. The wavelengths, $\lambda=0.58,0.78$, and $0.86 \mathrm{~m}$, have been selected to ensure the effects of surface tension to remain negligible, and the amplitudes of the background take the values $a=0.01,0.02$, and $0.03 \mathrm{~m}$. Figure 2 shows the measurements of surface elevation and phase.

It turns out that a stationary wall is separating two wave domains of weakly nonlinear waves with an identical amplitude and wave vector; however, fully inverted phases result. The domain wall is aligned with the propagation direction of the underlying Stokes waves. A photograph of one of the weakly nonlinear domain walls is shown in Fig. 3.

The measurements show very good agreement with the theoretical predictions from Eq. (9): The domain walls stretch out along the whole length of the tank without any noticeable change in properties.

We have also conducted some straightforward numerical simulations of the 2D + 1 NLS Eq. (4) using a (secondorder) finite difference scheme for the spatial discretization and a fourth-order Runge-Kutta method (with fixed time step) for the time marching.

Figure 4 shows the results for the reference case of lowest carrier wave steepness under study. Two different instants of time are depicted, $t=10$ and $100 \mathrm{~s}$. The numerical simulations suggest that for the time and length scales considered, the present domain wall pattern is not showing any sign of instability but remains stationary. We should stress at this point that transverse instability can be 


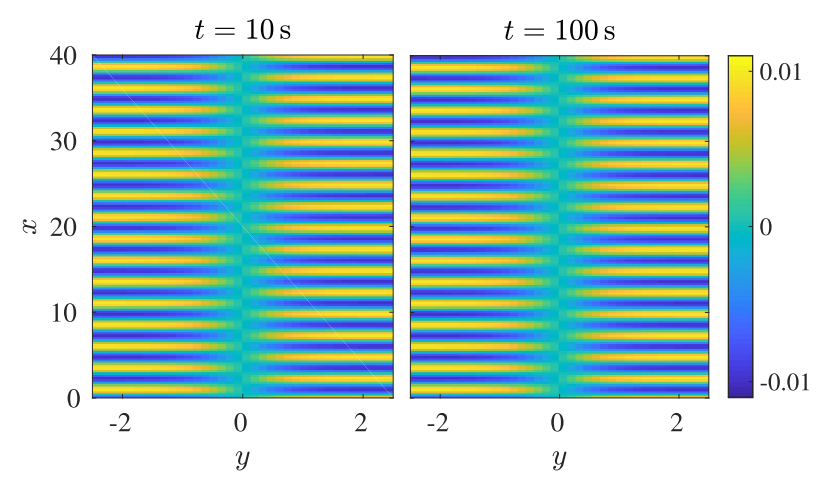

FIG. 4. Simulation results for $u(x, y)$ at $t=10$ and $100 \mathrm{~s}$. Parameters as in the top panel of Fig. $2: a=0.01 \mathrm{~m}, \kappa=10.8 \mathrm{~m}^{-1}$.

analyzed, when triggered following the application of a particular perturbation in the $y$ direction [36]. As our wave setup is well controlled, the spontaneous emergence and development of the transverse instability require significant time or length scales beyond our facility's configuration or dimensions.

We now investigate walls arising from general transformation angles $\gamma \neq \pi / 2$. The initial conditions for the wave paddles are again determined by Eq. (3). We investigate two cases: the first originates from the black soliton, $\theta=0$, and transformation angle $\gamma=0.4 \pi$. The second one starts from a gray soliton, $\theta=\pi / 6$, and a transformation angle $\gamma=0.35 \pi$.

Figure 5 shows the resulting analytical solution of the $2 \mathrm{D}+1$ NLS for the first case. Snapshots of the surface elevation are shown for subsequent times. In contrast to the previous solution, where the domain wall was oriented in the direction of the wave vector, now an oblique boundary results, which moves laterally but is still stationary in a moving reference frame. This is the general result also for other choices of parameters; a photograph is given in Fig. 6.

For a quantitative comparison between the solutions of the $2 \mathrm{D}+1 \mathrm{NLS}$ and the tank test, we took measurements along the wave tank at distances of $x=3,5,8$, and $10 \mathrm{~m}$ from the wave maker. The measurements are shown in the
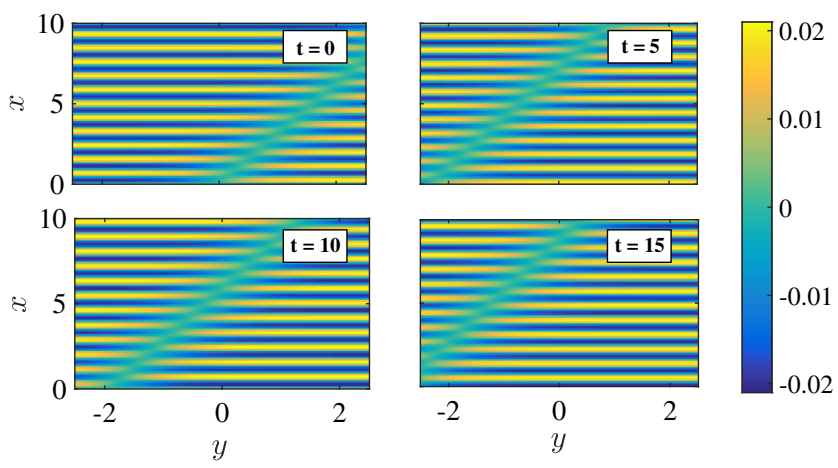

FIG. 5. Snapshots of the motion of a moving wall as resulting from Eq. (3) for four different times $t=0,5,10$, and $15 \mathrm{~s}$. $a=0.02 \mathrm{~m}, \kappa=7.3 \mathrm{~m}^{-1}, \chi=0, \theta=0$, and $\gamma=0.4 \pi$.

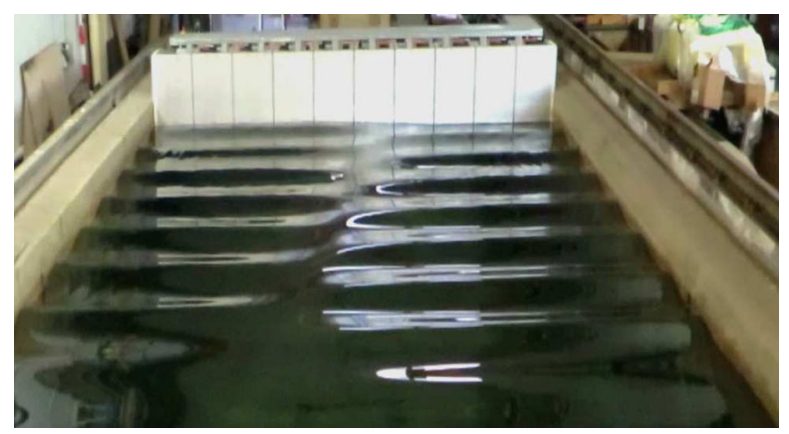

FIG. 6. Photographic image taken at a distance of $10 \mathrm{~m}$ from the wave maker for $a=0.02 \mathrm{~m}, \kappa=7.3 \mathrm{~m}^{-1}, \chi=0, \theta=0$, and $\gamma=0.4 \pi$.

left panels of Figs. 7 and 8 for $\theta=0$ and $\theta=\pi / 6$. When the domain wall passes the sensor locations, the amplitude experiences a depression as expected and corresponding well to the solution of the $2 \mathrm{D}+1 \mathrm{NLS}$. The experimental results agree very well with the new domain wall solutions of the $2 \mathrm{D}+1 \mathrm{NLS}$.

While the first observed domain wall solutions are stationary and oriented in the direction of the wave vector of the neighboring domains, the present oblique walls turn out to link neighboring wave domains with a phase difference of less than $\pi$, which comes at the expense of obliqueness and lateral motion of the domain wall.

To summarize our findings, we have demonstrated the existence of novel elementary types of domain wall solutions in the $2 \mathrm{D}+1 \mathrm{NLS}$. The new solutions suggest that weakly nonlinear domain walls, separating neighboring domains of weakly nonlinear waves with identical wave vector but differences in phase, do exist. For a phase difference of $\pi$ the domain walls are stationary and oriented along the wave vector of the neighboring domains. For other phase differences, the domain walls are obliquely oriented to the wave vector and stationary in laterally moving reference frames. An experimental illustration for
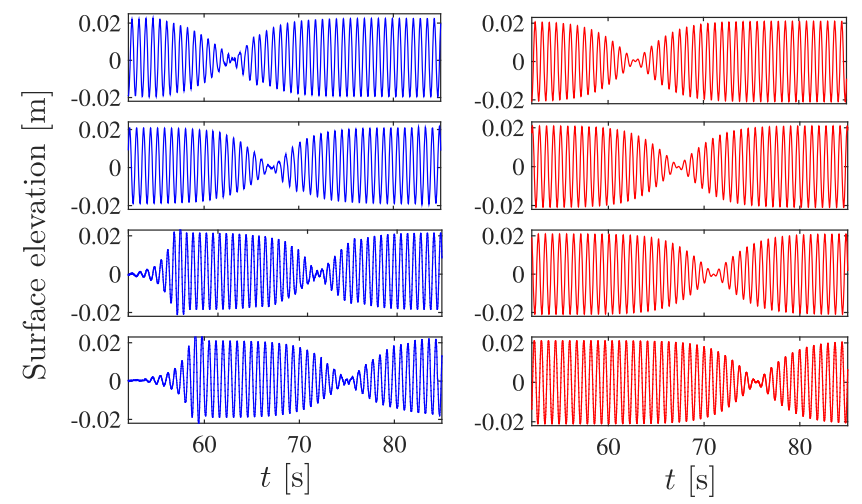

FIG. 7. Evolution of an oblique moving wall with $\theta=0$ and $\gamma=0.4 \pi$ for $a=0.02 \mathrm{~m}$ and $\kappa=7.3 \mathrm{~m}^{-1}$. Single point measurements (left) and corresponding theoretical predictions (right) at $x=3,5,8$, and $10 \mathrm{~m}$ from the wave maker. 

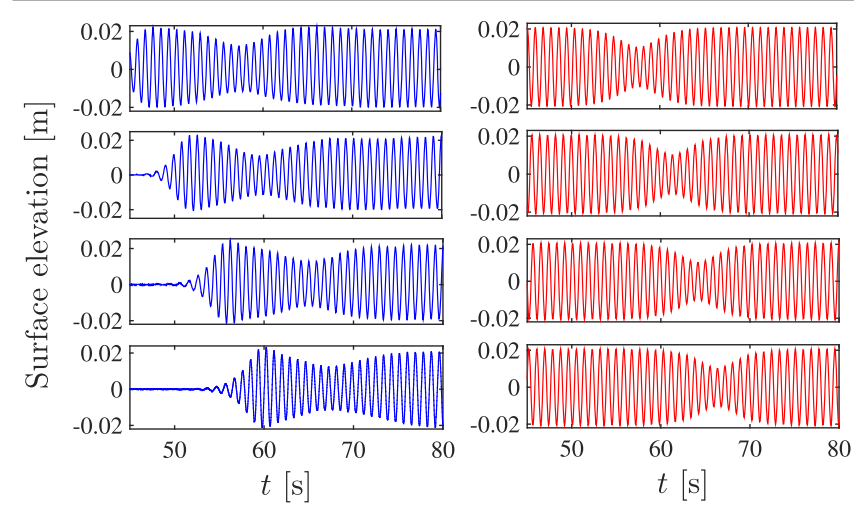

FIG. 8. Evolution of an oblique moving wall with $\theta=\pi / 6$ and $\gamma=0.35 \pi$ for $a=0.02 \mathrm{~m}$ and $\kappa=7.3 \mathrm{~m}^{-1}$. Single point measurements (left) and corresponding theoretical predictions (right) at $x=3,5,8$, and $10 \mathrm{~m}$ from the wave maker.

the solutions has been obtained for weakly nonlinear deep water surface gravity Stokes waves. For the nonlinearity range and the length and timescales considered, the measurements are in very good agreement with the predictions of the 2D +1 NLS theory.

Future work will be devoted to stability properties of the observed patterns. Further numerical studies will focus on limitations of the weakly nonlinear approach of the NLS in water waves. Moreover, similar studies in other universal fields of physics where weakly nonlinear dispersive waves arise may be motivated from the present findings.

The authors acknowledge support by Deutsche Forschungsgemeinschaft (DFG) Grant No. HO 3852/10.

[1] D. Y. Parpia, B. K. Tanner, and D. G. Lord, Nature (London) 303, 684 (1983).

[2] I. Aranson and L. Tsimring, Phys. Rev. Lett. 75, 3273 (1995).

[3] M. Gilles, P.-Y. Bony, J. Garnier, A. Picozzi, M. Guasoni, and J. Fatome, Nat. Photonics 11, 102 (2017).

[4] B. A. Malomed, A. A. Nepomnyashchy, and M. I. Tribelsky, Phys. Rev. A 42, 7244 (1990).

[5] B. A. Malomed, H. E. Nistazakis, D. J. Frantzeskakis, and P. G. Kevrekidis, Phys. Rev. A 70, 043616 (2004).

[6] B. A. Malomed, Phys. Rev. E 50, R3310(R) (1994).

[7] G. B. Whitham, Proc. R. Soc. A 283, 238 (1965).

[8] D. H. Peregrine, J. Fluid Mech. 136, 435 (1983).

[9] A. J. Roberts, J. Fluid Mech. 135, 301 (1983).

[10] A. J. Roberts and D. H. Peregrine, J. Fluid Mech. 135, 323 (1983).
[11] N. Akhmediev and V. I. Korneev, Theor. Math. Phys. 69, 1089 (1986).

[12] J. M. Dudley, F. Dias, M. Erkintalo, and G. Genty, Nat. Photonics 8, 755 (2014).

[13] D. M. Henderson, H. Segur, and J. D. Carter, J. Fluid Mech. 658, 247 (2010).

[14] D. M. Henderson, M. S. Patterson, and H. Segur, J. Fluid Mech. 559, 413 (2006).

[15] J. Hammack, D. M. Henderson, and H. Segur, J. Fluid Mech. 532, 1 (2005).

[16] V. E. Zakharov, J. Appl. Mech. Tech. Phys. 9, 190 (1968).

[17] D. J. Benney and A. C. Newell, J. Math. Phys. (Cambridge, Mass.) 46, 133 (1967).

[18] D. J. Benney and G. J. Roskes, Stud. Appl. Math. 48, 377 (1969).

[19] D. K. P. Yue and C.C. Mei, J. Fluid Mech. 99, 33 (1980).

[20] J. H. Pihl, C. C. Mei, and M. J. Hancock, Phys. Rev. E 66, 016611 (2002).

[21] A. V. Babanin and T. Waseda, Diffraction and Instability of Short-Crested Limited-Length One-Dimensional Coherent Wave Trains (ASME, St. John's, 2015).

[22] H. Hasimoto and H. Ono, J. Phys. Soc. Jpn. 33, 805 (1972).

[23] C. C. Mei, The Applied Dynamics Of Ocean Surface Waves, Advanced Series on Ocean Engineering 1 (Wiley-Interscience, 1983; World Scientific, 1989).

[24] A. Davey and K. Stewartson, Proc. R. Soc. A 338, 101 (1974).

[25] W. H. Hui and J. Hamilton, J. Fluid Mech. 93, 117 (1979).

[26] H. C. Yuen and B. M. Lake, Adv. Appl. Mech. 22, 67 (1982).

[27] P. G. Saffman and H. C. Yuen, Phys. Fluids 21, 1450 (1978).

[28] A. Shabat and V. Zakharov, J. Exp. Theor. Phys. 34, 1 (1972).

[29] N. Akhmediev and A. Ankiewicz, Solitons: Nonlinear Pulses and Beams (Chapman and Hall, London, 1997).

[30] A. Chabchoub, O. Kimmoun, H. Branger, N. Hoffmann, D. Proment, M. Onorato, and N. Akhmediev, Phys. Rev. Lett. 110, 124101 (2013).

[31] A. Chabchoub, O. Kimmoun, H. Branger, C. Kharif, N. Hoffmann, M. Onorato, and N. Akhmediev, Phys. Rev. E 89, 011002 (2014).

[32] D. J. Frantzeskakis, J. Phys. A 43, 213001 (2010).

[33] F. Baronio, S. Wabnitz, and Y. Kodama, Phys. Rev. Lett. 116, 173901 (2016).

[34] N. Masnadi and J. H. Duncan, J. Fluid Mech. 810, 448 (2017).

[35] A. V. Babanin, Breaking and Dissipation of Ocean Surface Waves (Cambridge University Press, Cambridge, England, 2011).

[36] Yu. S. Kivshar and D. E. Pelinovsky, Phys. Rep. 331, 117 (2000). 